\title{
Morphologie larvaire de Setaria labiatopapillosa (Nematoda, Filarioidea) chez Aedes aegypti
}

\author{
par Odile BAIN
}

Laboratoire de Zoologie (Vers) associé au C.N.R.S. ( $\mathrm{P}^{r}$ A.-G. CHABaud) du Museum National d'Histoire Naturelle, 57, rue Cuvier - F 75 - Paris ( $\left.5^{\circ}\right)$

et Section Entomologique, Section Onchocercose, Centre Muraz, Bobo-Dioulasso (Haute-Volta)

\begin{abstract}
Résumé
La morphologie larvaire de $S$. labiatopapillosa est précisée. Des caractères faciles à observer (longueur du corps, longueur de l'œsophage glandulaire et forme de la queue) permettent de distinguer les formes infectantes de cette espèce de celles de $S$. equina et de $S$. digitata.
\end{abstract}

\section{Summary}

A detailed morphology of the larvae of Setaria labiatopapillos $a$ is given. Some easily observed characters such as length of body, length of glandular œsophagus and shape of tail make possible the separation between the infestant forms of this species and those of $S$. equina and $S$. digitata.

\section{Introduction.}

Les grandes lignes du développement larvaire de Setaria labiatopapillosa (Alessandrini, 1938) chez Aedes aegypti ont été étudiées par Nelson (1962).

Nous donnons ici une description plus détaillée de la morphologie larvaire.

\section{Matériel expérimental.}

Un jeune veau a été infecté expérimentalement par une injection sous-cutanée de stades infectants. 
A. aegypti est une souche provenant de Kongolikan (Haute-Vo'ta), entretenue à la section entomologique du Centre Muraz.

Chaque $q$ gorgée prend en moyenne 14 microfilaires.

\section{Allure du développement.}

A $26^{\circ}-28^{\circ} \mathrm{C}$, les formes infectantes apparaissent onze jours après l'ingestion des microfilaires. Le développement s'effectue dans les muscles thoraciques; le premier stade dure quatre jours et demi, pendant lesquels la larve s'immobilise, s'organise et s'épaissit; la deuxième mue a lieu neuf jours et demi après le repas infectant; elle s'effectue chez des larves longues d'environ $1.800 \mu$., épaisses mais déjà mobiles; la maturation de la forme infectante dure deux jours environ et s'accompagne d'une croissance assez importante (corps long de $2.200 \mu$ à $2.600 \mu$ ).

\section{Description de la microfilaire. (fig. 1 A).}

Microfilaire à gaine; cuticule épaisse; tête ornée par deux demi-couronnes ventrales de pointes (fig. $1 \mathrm{G}$ ) et par un crochet subterminal gauche en forme d'écusson.

La coloration vita'e à l'Azur II met en évidence les noyaux rectaux et excréteurs, les pores anal et excréteur, ainsi que les deux noyaux des cellules musculaires qui actionnent le crochet céphalique; le corps interne, de taille variable, renferme 3 à 8 globules réfringents ou est constitué par une substance granuleuse; queue effilée.

Dimensions : corps long de $240 \mu$ à $310 \mu$ sur $6 \mu$ à $7,5 \mu$ de large (microfilaires mesurées dans le sang frais, après immobilisation par la chaleur); pour une microfilaire longue de $240 \mu$, les dimensions sont les suivantes (mensurations sur microfilaire colorée à l'Azur II) : noyaux des cellules musculaires du crochet céphalique à $10 \mu$ de l'apex; anneau nerveux, pore excréteur et noyau excréteur respectivement à $45 \mu, 70 \mu$ et $80 \mu$ de l'apex; corps interne long de $35 \mu$ et débutant à $115 \mu$ de l'apex; R1 et R4 respectivement à $160 \mu$ et $180 \mu$ de l'apex (les cellules rectales sont à peu près à égale distance 2 à 2); queue longue de $55 \mu$.

$\mathbf{1}^{\text {er }}$ stade larvaire (fig. 1 et 2 ).

- $18 \mathrm{~h}$ après le repas infectant (fig. $1 \mathrm{~B}$ ) : la larve est un peu moins mobile et légèrement épaissie dans la régıon postérieure; les noyaux pariétaux et les noyaux de la colonne nucléaire sont encore petits mais visibles sans coloration vitale; les quatre noyaux rectaux sont volumineux, ovales et plus proches du pore anal; celui-ci est fermé par une légère excroissance cuticulaire ; cinq petits noyaux ronds et vésiculeux apparaissent à l'ancien emplacement du corps interne; un axe chitineux va de l'apex de la microfilaire jusqu'au voisinage du plus antérieur de ces petits noyaux.

- $23 \mathrm{~h}$ après le repas infectant (fig. $1 \mathrm{C}$ ) : les noyaux rectaux R2 à R4 s'entourent de cytoplasme; les cellules R2 et R3 s'accolent au-dessus de R4 et se disposent symétriquement par rapport au plan sagittal de la microfilaire. Le noyau R1 est très volumineux. 
- $30 \mathrm{~h}$ à $40 \mathrm{~h}$ après le repas infectant (fig. $1 \mathrm{D}$ ) : les cytoplasmes des cellules R2 à $\mathrm{R} 4$ se sont soudés et forment un rectum triradié allongé ; la cellule R1 est divisée en deux cellules-filles ventrales; les cinq petits noyaux forment deux files de cellules intestinales (trois cellules sur le flanc gauche, deux cellules sur le flanc droit). La région postérieure de l'axe chitinoïde est entourée par un cône de cellu'es œsophagiennes dont la base est soudée à l'ébauche intestinale. La cellule excrétrice est volumineuse.

$-50 \mathrm{~h}$ après le repas infectant (fig. $1 \mathrm{E}$ et $\mathrm{F}$ ) : la larve est immobile; elle a une forme presque cylindrique, avec une queue qui se termine par une pointe très effilée et anucléée. Le tégument est formé de files de cellules volumineuses. Le rectum est presque rectangulaire. L'intestin et la base de l'œsophage sont fusionnés et forment un volumineux bourgeon. En arrière de la cellule excrétrice, un petit massif de cellules s'organise autour de l'axe chitinoïde; une troisième ébauche œsophagienne apparaît en avant de l'anneau nerveux.

Dimensions : $165 \mu$ de long et $30 \mu$ de large au maximum ; anneau nerveux, pore excréteur et début de l'ébauche œsophagienne postérieure respectivement à $40 \mu, 55 \mu$. et $70 \mu$ de l'apex ; ébauche intestinaie longue de $20 \mu$; rectum long de $30 \mu$; queue et pointe caudale longues respectivement de $35 \mu$ et $23 \mu$.

- $\mathrm{A}$ la fin du troisième jour (fig. $1 \mathrm{~A}$ et $\mathrm{B}$ ) : la larve est plus épaisse et l'œsophage est entièrement formé par deux ensembles distincts : un massif postérieur et un cordon antérieur; lintestin commence à se creuser; les massifs de cellules ganglionnaires s'individualisent.

- $95 \mathrm{~h}$ après le repas infectant (fig. $2 \mathrm{C}$ ) : la fin du stade I se caractérise par une multiplication des cellules intestinales et œsophagiennes et par l'apparition d'une constriction entre l'intestin et l'œsophage.

Dimensions : $215 \mu$ de long et $40 \mu$ de large au maximum ; anneau nerveux et pore excréteur respectivement à $38 \mu$ et $60 \mu$ de l'apex; œsophage et intestin longs respectivement de $98 \mu$ et $40 \mu$; rectum long de $38 \mu$; queue et pointe caudale longues respectivement de $40 \mu$ et $22 \mu$.

- Quatre jours et demi après le repas infectant (mue I) (fig. $2 \mathrm{D}$ et $\mathrm{E}$ ) : la cuticule du premier stade est décollée aux extrémités du corps; l'apex de l'axe chitinoïde œsophagien est entouré de grosses cellu'es pharyngiennes; l'intestin s'est allongé et creusé ; le bouchon cuticulaire anal est très saillant, il est strié près des cellules rectales ; l'ébauche génitale est située à mi-hauteur de l'intestin.

Dimensions : $260 \mu$ de long et $38 \mu$ de large ; anneau nerveux et pore excréteur respectivement à $45 \mu$ et $75 \mu$ de l'apex; œsophage et intestin longs respectivement de $110 \mu$ et $80 \mu$; queue longue de $35 \mu$ avec pointe caudale de $5 \mu$.

2 stade larvaire (fig. 3 et fig. 4 A et B).

La larve du deuxième stade a une capsule buccaie en forme d'entonnoir ; la bouche est comprimée latéralement, bordée par quatre papilles (fig. 3 D) et deux grandes amphides; quatre massifs de cellules ganglionnaires sont appendus à l'anneau nerveux : un massif ventral qui descend un peu au-dessous de la cellule excrétrice, un massif dorsal très court et deux massifs latéraux. L'œsophage se termine par une valvule. 

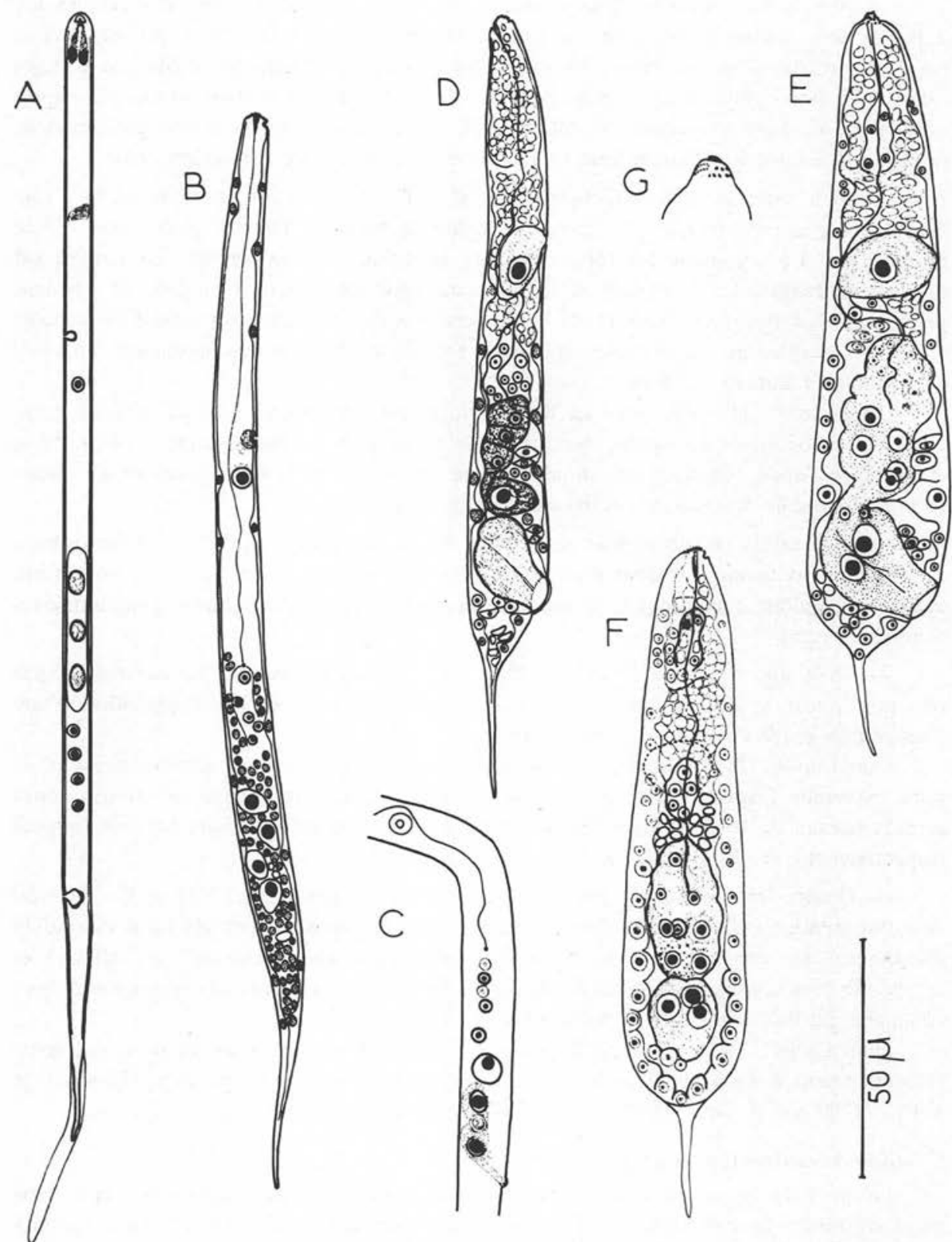

FIg. 1. - 1 $1^{\text {er }}$ stade larvaire: A. : microfilaire après coloration vitale, vue latérale gauche. B. : $18^{\mathrm{e}} \mathrm{h}$, vue ventrale. - C.: $23^{\mathrm{e}} \mathrm{h}$, régions rectale et intestinale et fin de l'axe cuticulaire œsophagien. - D. : $40^{\circ} \mathrm{h}$. - E. et F. : $50^{\circ} \mathrm{h}$, respectivement en vue latérale droite et en vue dorsale (éch. $50 \mu$, sauf G., à main levée) 

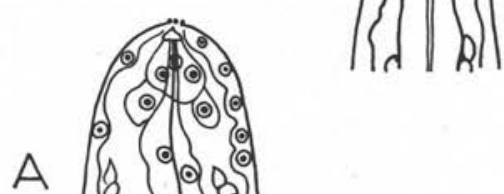

A

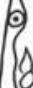

(2) (-)

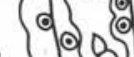

.

0

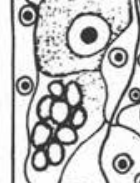

()

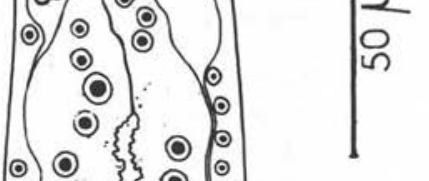

- 0 ○

$\odot$

$\circ$

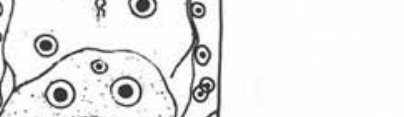

(2)

B

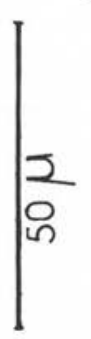

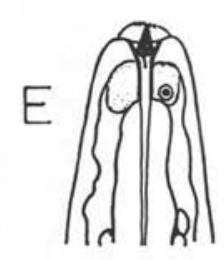

C
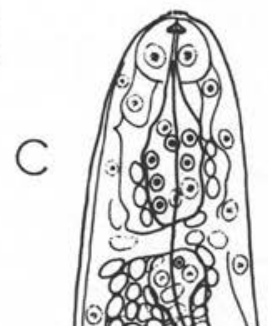

6

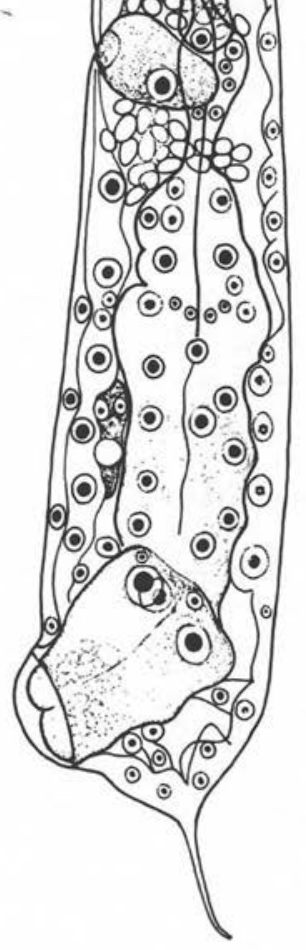

D

(2)
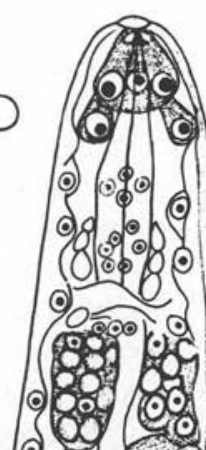

ब)

2

d).

$\circ 80$

$0^{\circ}$

(ㅇ)

c)

d)

$2 \%$

0. 0.130

๑) 0.100

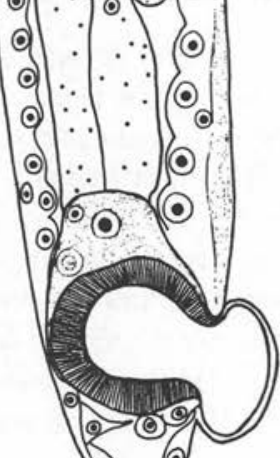

ino

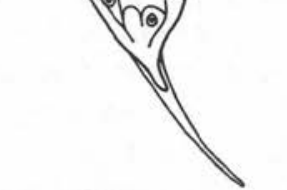

Fig. 2. - A. à C. : $1^{\text {er }}$ stade larvaire à la $95^{\mathrm{e}} \mathrm{h} .-\mathrm{A}$. : vue dorsale. - B. : bouchon anal vu de face. - C. : vue latérale gauche. - D.: mue I, à 4 jours $1 / 2$. - E. : idem, détail de la région céphalique (éch. $50 \mu$ ) 
L'intestin a une paroi amincie et renferme les déchets du métabolisme. Le rectum est formé de trois volumineuses cellules au cytoplasme granuleux (une dorsale et deux latéro-ventrales); la jonction de l'intestin et du rectum est réalisée par deux cellules de même texture que les ceilules rectales. Le tégument est très épais.

$\mathrm{Au}$ cours du deuxième stade, les noyaux de l'œsophage se multiplient en arrière de l'anneau nerveux et forment l'œsophage glandulaire (fig. $3 \mathrm{~A}$ et B). La cellule excrétrice se prolonge latéralement par deux larges bandes transversales. L'ébauche génitale est au niveau de l'intestin chez la larve $\sigma^{*}$ (fig. 3 B) et en arrière de la cellule excrétrice chez la larve . La pointe caudale est fréquemment atrophiée.

Dimensions d'une larve de six jours et demi (fig. $3 \mathrm{~A}$ à D) (larve $\delta^{*}$ ) : $640 \mu$ de long et $40 \mu$ de large ; capsule buccale haute de $12 \mu$; anneau nerveux et pore excréteur respectivement à $65 \mu$ et $95 \mu$ de l'apex; œsophage musculaire, œsophage glandulaire et intestin longs respectivement de $90 \mu, 145 \mu$ et $350 \mu$; rectum long de $48 \mu$. et large de $38 \mu$; queue longue de $30 \mu$.

Plus tard, la larve devient bien mobile et s'allonge rapidement $(1.400 \mu$ le huitième jour) (fig. $3 \mathrm{E}$ et $\mathrm{F}$ et fig. $4 \mathrm{~A}$ ); l'œsophage musculaire s'allonge et se différencie; l'œsophage glandulaire renferme de très nombreux noyaux, mais les grains de sécrétion sont encore rares; le bouchon cuticulaire anal reste très volumineux.

- Neuf jours et demi après le repas infectant, a lieu la mue II ; une capsule buccale très courte se forme en arrière de la capsule du deuxième stade chez des spécimens mesurant environ $1.800 \mu$. (fig. 4 B); l'œsophage glandulaire se charge de granulations; le bouchon anal est expulsé ainsi que les déchets contenus dans l'intestin.

\section{Maturation de la forme infectante. (fig. $4 \mathrm{C}$ à $\mathrm{I}$ ).}

Après la mue II, la larve subit diverses modifications: elle s'allonge encore, elle s'amincit (le tégument est moins épais) et les structures cellulaires s'estompent ; l'œsophage glandulaire acquiert une structure grenue; la queue s'affine, les cellules rectales s'étirent, la mobilité s'accentue.

Chez la larve infectante mûre (au douzième jour) (fig. $4 \mathrm{C}$ et $\mathrm{I}$ ), la tête porte huit papilles qui se rapprochent des axes latéraux et deux amphides; les papilles internes sont pointues. La bouche et la cavité buccale sont comprimées latéra'ement ; la capsule buccale est réduite à un court rhabdion sclérifié.

Déirides exiguës au niveau de la cellule excrétrice.

La cellule excrétrice est à mi-hauteur entre l'anneau nerveux et le début de l'œsophage glandulaire; le canai excréteur est assez long et se termine dans un sinus bien cuticularisé; en arrière de la cellu'e excrétrice se trouvent deux masses ganglionnaires ventrales (fig. $4 \mathrm{E}$ ) et l'ébauche génitale 9 . Chez le ơ', l'ébauche génitale est située dans la région antérieure de l'intestin (fig. $4 \mathrm{D}$ ). Les parois de l'intestin sont très épaisses (fig. $4 \mathrm{D}$ et $\mathrm{H}$ ).

Le rectum comprend une portion tubulaire distale et les trois cellules rectales proximales. La queue est légèrement incurvée ventralement et se termine par une pointe 

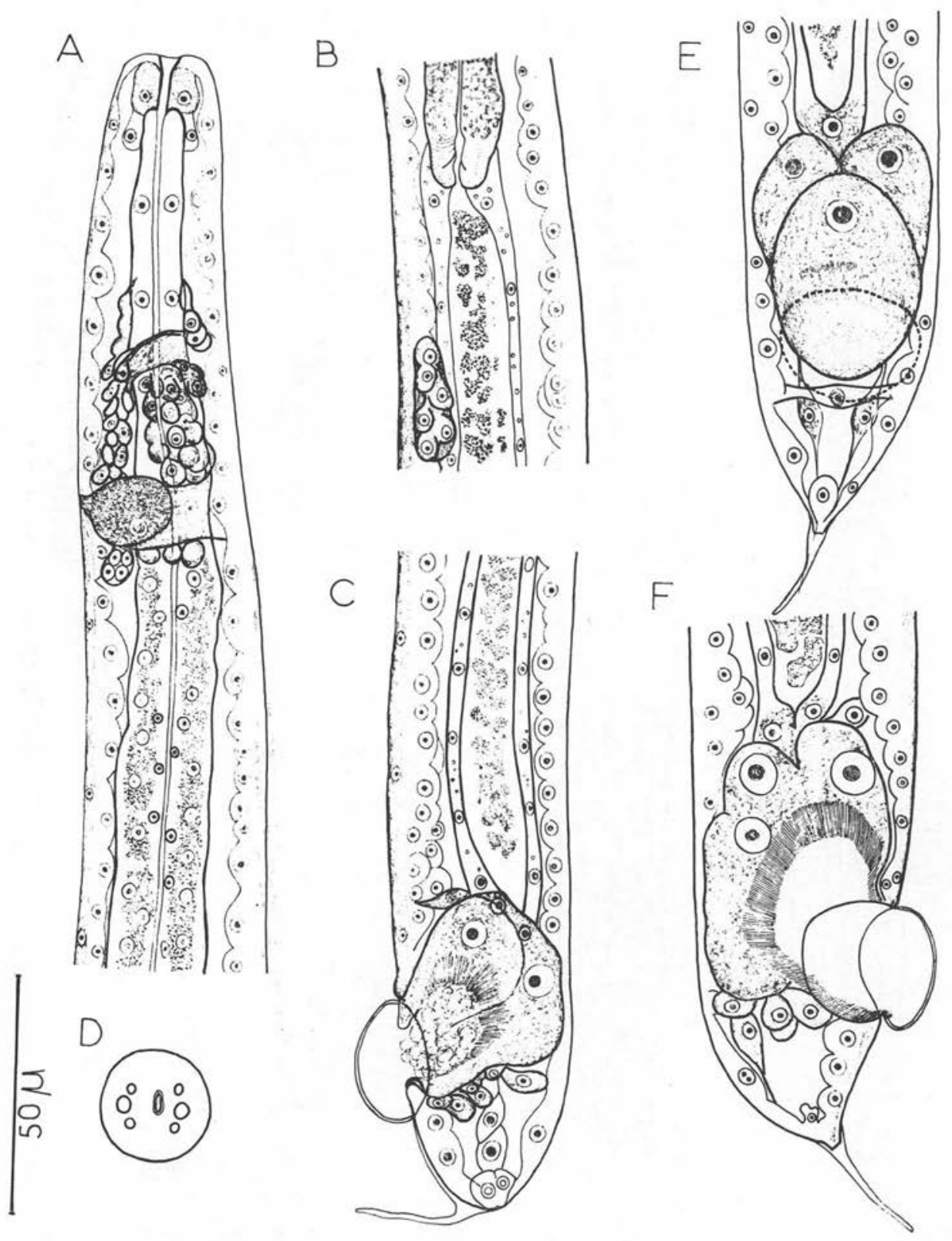

FIG. 3. $-2^{e}$ stade larvaire. - A. à D. : larve $\sigma^{\star}$ de 6 jours $1 / 2$. - A. : région antérieure. B. : région intestinale au niveau de l'ébauche génitale. - C. : région caudale en vue latérale. D. : tête en vue apicale d'un stade II. - E. et F. : région caudale d'un stade II âgé de 8 jours en vues dorsale et latérale (éch. $50 \mu$ ) 


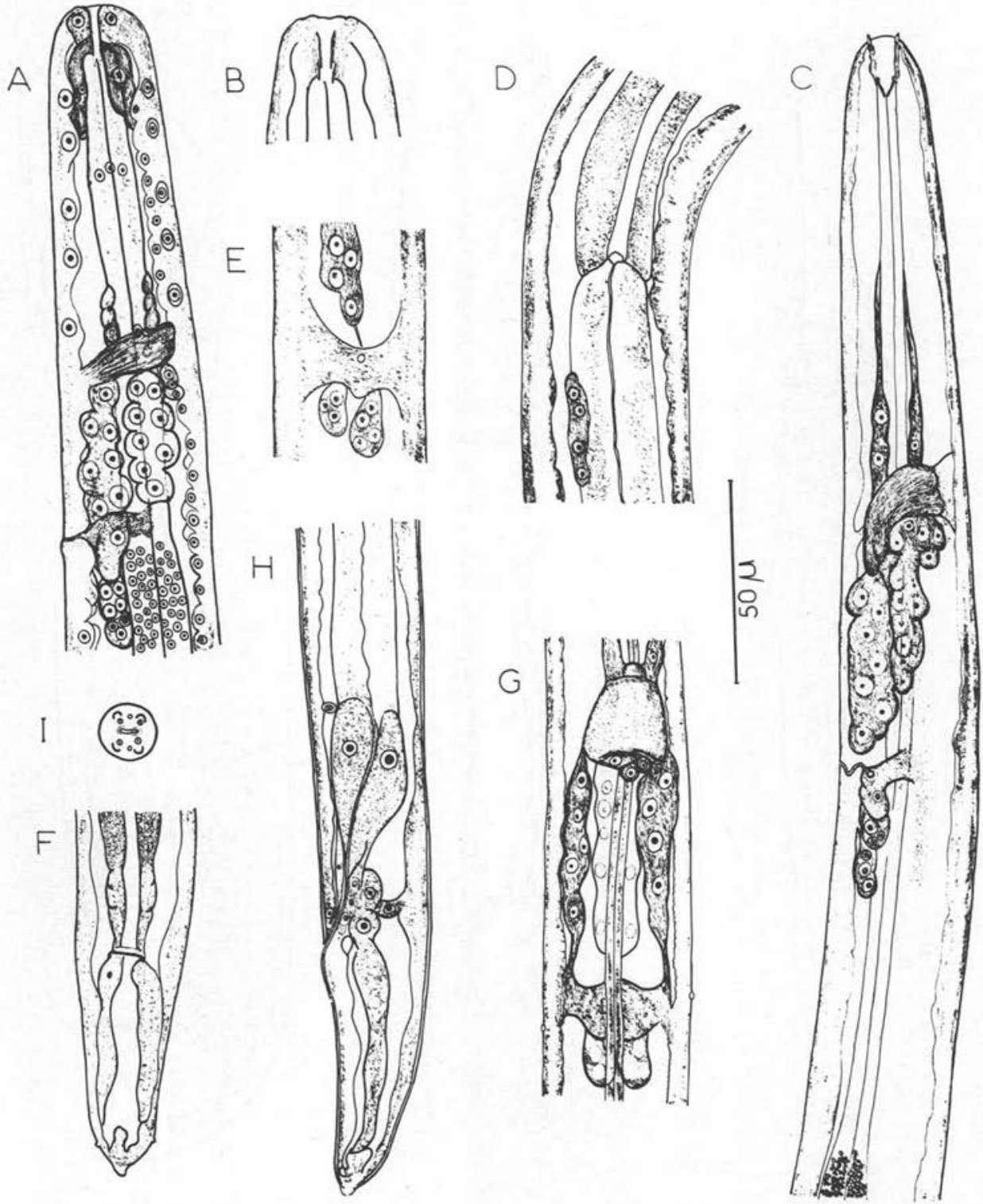

FIG. 4. - A. : région antérieure d'un stade II $q$ âgé de 8 jours. - B. : région céphalique d'une larve en mue II, à 9 jours $1 / 2$. - C. à I. : stade infectant. - C. : région antérieure d'une larve 0. - D.: position de l'ébauche génitale chez une larve $\delta$. - E. : région du pore excréteur chez une larve $\delta$, vue ventrale. - F.: région caudale, vue ventrale. - G. : disposition des masses ganglionnaires chez la larve $\delta$, vue dorsale. - H. : région caudale, vue latérale. - I. : tête, vue apicale (éch. $50 u$ ) 
obtuse ; deux mucrons subterminaux ornent la queue latéralement (parfois l'un d'entre eux est atrophié) (fig. $4 \mathrm{~F}$ et $\mathrm{H}$ ).

Dimensions (mensurations effectuées sur une larve infectante vivante, immobilisée par la chaleur) : longueur variant de $2.200 \mu$ à $2.600 \mu$; corps large de $40 \mu$ à $42 \mu$. Sur le spécimen dessiné, cavité buccale haute de $15 \mu$, anneau nerveux et pore excréteur situés respectivement à $110 \mu$ et $185 \mu$ de l'apex; œsophages musculaire et glandulaire longs respectivement de $270 \mu$ et $1.460 \mu$; rectum et queue longs respectivement de $62 \mu$ et $55 \mu$.

\section{Conclusion.}

S. labiatopapillosa, Filaire de la famille Setariidae, a un développement larvaire analogue à celui des Onchocercidae.

Les cycles de $S$. digitata (v. Linstow, 1906) effectués par Itagaki et Taniaguchi (1954) et repris par Ono (1960), et de S. equina (Abildgaard, 1789) effectué par ce deuxième auteur (1960), montrent que les larves de ces trois espèces se développent dans les muscles thoraciques de divers Culicides.

Leurs formes infestantes présentent des caractères qui permettent de les discerner entre elles : $S$. equina est plus petite que les deux autres espèces $(1.500 \mu-1.800 \mu)$ et son extrémité caudale porte un processus hyalin terminal, en plus des deux petites languettes latéro-ventrales subterminales communes aux trois espèces: $S$. labiatopapillosa et $S$. digitata sont grandes (respectivement $3.200 \mu-2.600 \mu$ et $2.000 \mu-2.400 \mu$ ), mais la première espèce a un œsophage glandulaire très long $(1.400 \mu$ à $1.700 \mu)$ et sa queue se termine par une grosse pointe conique tandis que la queue est simplement arrondie chez $S$. digitata.

\section{Bibliographie}

Itagaki (S.) et Taniaguchi (M.), 1954, in Nelson, 1962. - Pathogenicity of $S$. digitata against domestic animals (Sheep, Goats and Horses) and its life cycle. Jap. J. San. Zoology., 4, special $\mathrm{n}^{\circ}$ comm. 70th Birthday of Dr. H. Kobayashi (ouvrage non consulté).

NeLson (G.S.), 1962. - Observations on the development of Setaria labiatopapillosa using new techniques for infecting Aedes aegypti with this Nematode. J. Helminth., 36, p. 281-296.

ONo (N.), 1960. - Comparative studies on various stages of Filariid larvae of Setaria sp. in anophelin mosquitoes. J. Kurume. Med. Ass., 23, p. 1360-1378. 